\title{
The solar photospheric abundance of europium
}

\section{Results from CO5BOLD 3D hydrodynamical model atmospheres}

\author{
A. Mucciarelli ${ }^{1}$, E. Caffau ${ }^{2}$, B. Freytag ${ }^{3}$, H.-G. Ludwig ${ }^{2, \star}$, and P. Bonifacio ${ }^{2,4, \star}$ \\ 1 Dipartimento di Astronomia, Università degli Studi di Bologna, Via Ranzani 1, 40127 Bologna, Italy \\ e-mail: alessio.mucciarelli@studio.unibo.it \\ 2 GEPI, Observatoire de Paris, CNRS UMR 8111, Université Paris Diderot, Place Jules Janssen, 92190 Meudon, France \\ e-mail: [elisabetta.caffau; hans.ludwig; piercarlo.bonifacio]@obspm.fr \\ 3 Centre de Recherche Astrophysique de Lyon, UMR 5574 CNRS, Université de Lyon, École Normale Supérieure de Lyon, \\ 46 allée d'Italie, 69364 Lyon Cedex 07, France \\ e-mail: Bernd.Freytag@ens-lyon.fr \\ ${ }^{4}$ Istituto Nazionale di Astrofisica, Osservatorio Astronomico di Trieste, Via Tiepolo 11, 34143 Trieste, Italy \\ Received 25 December 2007 / Accepted 6 March 2008
}

\section{ABSTRACT}

\begin{abstract}
Context. Europium is an almost pure $r$-process element, which may be useful as a reference in nucleocosmochronology. Aims. Determine the photospheric solar abundance using CO5BOLD 3D hydrodynamical model atmospheres.

Methods. Disc-centre and integrated-flux observed solar spectra are used. The europium abundance is derived using equivalent-width measurements. As a reference, one-dimensional model atmospheres are in addition used.

Results. The europium photospheric solar abundance $(0.52 \pm 0.02)$ agrees with previous determinations. We determine the photospheric isotopic fraction of ${ }^{151} \mathrm{Eu}$ to be $49 \% \pm 2.3 \%$ using the intensity spectra, and $50 \% \pm 2.3 \%$ using the flux spectra. This compares well to the meteoritic isotopic fraction $47.8 \%$. We explore 3D corrections for dwarfs and sub-giants in the temperature range $~ 5000 \mathrm{~K}$ to $\sim 6500 \mathrm{~K}$ and solar and 1/10-solar metallicities and find them to be negligible for all models investigated.

Conclusions. Our photospheric Eu abundance agrees well with previous determinations based on 1D models. This is in line with our conclusion that 3D effects for this element are negligible in the case of the Sun.
\end{abstract}

Key words. Sun: abundances - stars: abundances - hydrodynamics

\section{Introduction}

Europium $(Z=63)$ is formed by neutron captures on to seednuclei. In a seminal paper by Burbidge et al. (1957), it was assigned both to the $s$-process (slow neutron capture) and to the $r$-process (rapid neutron capture). According to current understanding about $r$-process nucleosynthesis, europium is an almost pure $r$-process element. About $95-97 \%$ of the $\mathrm{Eu}$ in the solar system is produced by the $r$-process (Arlandini et al. 1999; Burris et al. 2000). See the introduction in the paper by Mashonkina \& Gehren (2000) on the significance of the Eu/Ba ratio for assessing the relative contributions of the $r$-process and $s$-process.

The trend of the $[\mathrm{Eu} / \mathrm{Fe}]$ abundance ratio as a function of $[\mathrm{Fe} / \mathrm{H}]$ in the Milky Way stars, both halo and disk, appear to follow a similar behaviour to the $[\alpha / \mathrm{Fe}]$ ratio, which has an enhanced value in metal-poor stars and a decrease for $[\mathrm{Fe} / \mathrm{H}]>-1$ dex down to solar values. Measurements of this abundance ratio in extra-galactic stars highlight a decoupling between $[\mathrm{Eu} / \mathrm{Fe}]$ and $[\alpha / \mathrm{Fe}]$ ratios, with over-solar $[\mathrm{Eu} / \mathrm{Fe}]$ at high metallicity and solar or sub-solar $[\alpha / \mathrm{Fe}]$ values, as observed in Sagittarius (Bonifacio et al. 2000), in the Small Magellanic Cloud (Hill 1997), and the Large Magellanic Cloud (Hill et al. 1995).

The ratio of the two $r$-process elements $\mathrm{Eu} / \mathrm{Th}^{1}$ is potentially an interesting chronometer, provided that the production ratio of

\footnotetext{
* CIFIST, Marie Curie Excellence Team.

1 The isotope ${ }^{232} \mathrm{Th}$ is radioactive with a half-life of $14.05 \mathrm{Gyr}$.
}

the two nuclei can be predicted reliably theoretically (see Cowan $\&$ Sneden 2004, for an extensive review of the $r$-process).

In this paper we reconsider the Eu solar abundance in the light of the recent progress of the 3-dimensional (hereafter 3D) model atmosphere computations, measuring the solar abundance both with 1-dimensional (1D) and 3D models in order to assess the impact of this new generation of models on the solar Eu abundance.

The Eu solar abundance reported in the compilation of Grevesse \& Sauval (1998) is $A(\mathrm{Eu})=0.51 \pm 0.08^{2}$ and $A(\mathrm{Eu})=$ $0.55 \pm 0.02 \mathrm{dex}$, from the solar photosphere and from meteorites, respectively. The analysis of Lawler et al. (2001) yields $A(\mathrm{Eu})=0.52 \pm 0.01 \mathrm{dex}$, adopting new atomic data for the Eu lines and taking into account the hyperfine structure of the lines employed.

\section{Solar europium chemical analysis}

\subsection{Theoretical tools}

We derive the solar $\mathrm{Eu}$ photospheric abundance using $3 \mathrm{D}$ model atmospheres computed using the $\mathrm{CO}^{5} \mathrm{BOLD}$ code (COnservative COde for the COmputation of COmpressible COnvection in a BOx of $L$ Dimensions with $L=2,3$ ) (Freytag et al. 2002; Wedemeyer et al. 2004) and we compare our results to $1 \mathrm{D}$ model results. $\mathrm{CO}^{5} \mathrm{BOLD}$ solves the coupled non-linear equations of compressible hydrodynamics including

\footnotetext{
${ }^{2}$ We adopt the spectroscopic notation $A(\mathrm{X})=\log (N(\mathrm{X}) / N(\mathrm{H}))+12$.
} 
non-local frequency-dependent radiation transport for a small volume located at the stellar surface (for technical issues, see the on-line manual available at http://www.astro.uu.se/ bf/ co5bold/index.html). The atmospheric-flow field is sampled in equal temporal intervals each of which we call a "snapshot". In total, 25 snapshots were selected from a $\mathrm{CO}^{5} \mathrm{BOLD}$ simulation to represent the solar photosphere.

As references, we also adopted several 1D solar models:

- the 1D solar model computed by F. Castelli ${ }^{3}$ using the ATLAS9 code, adopts the solar abundances of Asplund et al. (2005), an $T_{\text {eff }}=5777 \mathrm{~K}, \log g=4.4377$, and microturbulence velocity of $1 \mathrm{~km} \mathrm{~s}^{-1}$;

- the semi-empirical solar atmosphere model derived by Holweger \& Müller (1974);

- a solar 1D atmospheric model obtained using the temporal and horizontal average of the 3D structure over surfaces of equal Rosseland optical depth. The comparison between the $3 \mathrm{D} \mathrm{CO}^{5} \mathrm{BOLD}$ model and this kind of average 3D model, allows us to estimate the influence of the fluctuations, around the mean stratification, on the line formation process;

- a solar 1D atmospheric model computed using a Lagrangian hydrodynamical code LHD (see Caffau \& Ludwig 2007), which includes the same opacities and equation-of-state adopted by the CO ${ }^{5}$ BOLD $3 \mathrm{D}$ code. LHD code treats convection using the standard mixing-length theory (MLT) in the formulation given by Mihalas (1978). The use of this kind of models allows us to compare these 1D models directly with the $3 \mathrm{D} \mathrm{CO}^{5} \mathrm{BOLD}$ models, which erases the systematics due to different physical assumptions.

The spectral synthesis from ATLAS and HM models are performed using the SYNTHE code (Kurucz 1993, 2005) in its Linux version (Sbordone et al. 2004; Sbordone 2005). For $\mathrm{CO}^{5} \mathrm{BOLD}$ and LHD models, the Linfor $3 \mathrm{D}^{4}$ code is used.

\subsection{Observational material}

The present study is based on two sets of high-resolution, high signal-to-noise ratio spectra of solar-flux and disc-centre intensity:

- Solar Flux - We adopted the solar-flux spectra of Neckel \& Labs (1984) and of Kurucz (2005) .

- Solar Intensity - As centre-disc solar-intensity spectra, we used the intensity of Neckel \& Labs (1984) and that of Delbouille et al. (1973) 6 .

We selected 5 Eu II optical spectral lines from the list of Lawler et al. (2001). It is worth noting that not all of these solar spectra are useful to measure the selected features. In the following, we describe briefly the single Eu II features used in this analysis and the corresponding adopted solar spectrum:

- $412.972 \mathrm{~nm}$ - strong Eu II line, with a weak blending on the blue side; this feature exhibits the same shape in all adopted solar spectra, without telluric contamination;

\footnotetext{
${ }^{3}$ http://www.user.oats.inaf.it/castelli/sun/ ap00t5777g44377k1asp.dat

${ }^{4}$ http://www.aip.de/ mst/Linfor3D/linfor_3D_manual. $\operatorname{pdf}$

5 See http://kurucz.harvard.edu/sun.html

${ }^{6}$ http://bass2000.obspm.fr/solar_spect.php
}

Table 1. Atomic data for the europium lines considered in this work.

\begin{tabular}{ccc}
\hline \hline $\begin{array}{c}\text { Wavelength } \\
(\mathrm{nm})\end{array}$ & $\log g f$ & $\begin{array}{c}E . P . \\
(\mathrm{eV})\end{array}$ \\
\hline 412.972 & 0.22 & 0.000 \\
604.951 & -0.80 & 1.278 \\
664.510 & 0.12 & 1.379 \\
707.709 & -0.72 & 1.249 \\
721.756 & -0.35 & 1.229 \\
\hline
\end{tabular}

- $604.951 \mathrm{~nm}$ - clean feature without particular difficulties, it is not blended or contaminated by nearby lines to cause problems in the placement of the continuum;

- $664.519 \mathrm{~nm}$ - this is one of the strongest optical transitions and is commonly used to infer the Eu II abundance. In all four of the solar observed spectra we consider this line results not blended with telluric features. This feature has a blending in its red wing, due to the presence of the weak features of $\mathrm{Cr}$ I and Si I;

- $707.709 \mathrm{~nm}$ - this weak line shows an $\mathrm{H}_{2} \mathrm{O}$ telluric-line contamination on its red side and only in the Delbouille spectrum does this blending seems to be less severe;

- $721.756 \mathrm{~nm}$ - this line is measurable only in the Delbouille spectrum; the other solar spectra show a strong blending of this feature with a $\mathrm{H}_{2} \mathrm{O}$ telluric-line, which is completely absent in the Delbouille spectrum.

\subsection{Chemical analysis}

\subsubsection{Analysis}

The chemical analysis of the selected Eu II features was performed adopting the atomic parameters for the Eu II lines by Lawler et al. (2001) and is summarised in Table 1. The Eu II spectral lines display significant hyperfine structures. We include in the line list, hyperfine structure and isotopic splitting, using the meteoritic isotopic ratio ${ }^{7}$ and the hyperfine constants A and B measured by Lawler et al. (2001). The calculation of the hyperfine structure was completed using the code LINESTRUC, described by Wahlgren (2005). All the hyperfine components for each Eu II feature, computed without the assumption of a specific isotopic ratio, are available in Table 4. We did not take into account possible NLTE effects, which are different between intensity and flux spectra and could explain the small positive difference between intensity and flux abundances. Mashonkina (2000) analysed the NLTE effects for the resonance Eu II line at $421.9 \mathrm{~nm}$ in solar-like stars, finding a NLTE correction of $\sim 0.04$ dex.

The solar Eu abundance was derived from the curve of growth of each line calculated using Linfor3D, adopting a meteoritic isotopic ratio. The equivalent width $(E W)$ of the Eu II lines was measured using a Gaussian fit with the IRAF task SPLOT, for its deblending option. The 3D models include only the Eu II lines, without the contribution of possible blending features. The choice to infer the abundance using the $E W$ measurement was made because of the inefficiency of the line-profile fitting with a 3D grid, due to the lack of the weak-blending components in the $3 \mathrm{D}$ synthetic spectra. This is caused by the inability of the current version of Linfor3D to handle a large number of lines. In Table 2, we provide our results for both

\footnotetext{
7 This element has two isotopes, with $Z=151$ and $Z=153$ with meteoritic abundance of $47.8 \%$ and $52.2 \%$ respectively (Anders \& Grevesse 1989).
} 
Table 2. Solar europium abundances from the adopted observed spectra. Column (1) indicates the solar spectrum used: KF: Kurucz flux, NF: Neckel flux, NI: Neckel intensity, DI: Delbouille intensity. Column (2) is the wavelength of the observed lines. Column (3) is the Equivalent Width. Columns (4) and (5) are Eu abundance from the $\mathrm{CO}^{5} \mathrm{BOLD}$ models and the corresponding uncertainty due to the error of the Equivalent Width. Columns (6)-(9) are the results from the 1D chemical analysis by adopting two solar models (HM: Holweger \& Müller (1974). FC: ATLAS9 solar model by Fiorella Castelli). Finally, Cols. (10) and (11) are the 3D corrections.

\begin{tabular}{|c|c|c|c|c|c|c|c|c|c|c|}
\hline Spec & $\begin{array}{l}\text { Wave } \\
\text { nm }\end{array}$ & $\begin{array}{l}E W \\
(\mathrm{pm})\end{array}$ & $\begin{array}{c}3 \mathrm{D} \\
\mathrm{CO}^{5} \mathrm{BOLD} \\
(\mathrm{dex})\end{array}$ & $\begin{array}{l}\sigma_{E W} \\
(\operatorname{dex})\end{array}$ & $\begin{array}{c}\text { 1D } \\
\text { HM } \\
(\operatorname{dex})\end{array}$ & $\begin{array}{c}N\left({ }^{151} \mathrm{Eu}\right) / N\left(\mathrm{Eu}_{\mathrm{tot}}\right) \\
\mathrm{HM}\end{array}$ & $\begin{array}{c}1 \mathrm{D} \\
\mathrm{FC} \\
(\operatorname{dex})\end{array}$ & $\begin{array}{c}N\left({ }^{151} \mathrm{Eu}\right) / N\left(\mathrm{Eu}_{\mathrm{tot}}\right) \\
\mathrm{FC}\end{array}$ & $\begin{array}{l}\text { 3D-1D } \\
\qquad(\mathrm{dex})\end{array}$ & $\begin{array}{c}3 D-\langle 3 D\rangle \\
(\operatorname{dex})\end{array}$ \\
\hline KF & 412.972 & 5.620 & 0.509 & 0.045 & 0.535 & 0.54 & 0.526 & 0.51 & -0.013 & -0.030 \\
\hline $\mathrm{NF}$ & 412.972 & 5.652 & 0.513 & 0.045 & 0.527 & 0.49 & 0.513 & 0.48 & -0.014 & -0.030 \\
\hline NI & 412.972 & 5.026 & 0.537 & 0.050 & 0.533 & 0.51 & 0.537 & 0.49 & 0.014 & 0.006 \\
\hline DI & 412.972 & 5.045 & 0.540 & 0.050 & 0.545 & 0.53 & 0.541 & 0.51 & 0.015 & 0.006 \\
\hline $\mathrm{KF}$ & 604.951 & 0.063 & 0.504 & 0.030 & 0.528 & - & 0.539 & - & 0.013 & 0.002 \\
\hline $\mathrm{NF}$ & 604.951 & 0.061 & 0.490 & 0.030 & 0.519 & - & 0.492 & - & 0.014 & 0.003 \\
\hline NI & 604.951 & 0.051 & 0.480 & 0.035 & 0.532 & - & 0.487 & - & 0.023 & 0.013 \\
\hline DI & 604.951 & 0.057 & 0.529 & 0.035 & 0.537 & - & 0.521 & - & 0.022 & 0.012 \\
\hline $\mathrm{KF}$ & 664.519 & 0.436 & 0.511 & 0.020 & 0.540 & 0.52 & 0.520 & 0.51 & 0.011 & 0.000 \\
\hline $\mathrm{NF}$ & 664.519 & 0.432 & 0.507 & 0.020 & 0.531 & 0.49 & 0.520 & 0.47 & 0.011 & 0.000 \\
\hline NI & 664.519 & 0.400 & 0.530 & 0.026 & 0.506 & 0.46 & 0.518 & 0.47 & 0.020 & 0.010 \\
\hline DI & 664.519 & 0.384 & 0.514 & 0.021 & 0.504 & 0.50 & 0.504 & 0.49 & 0.020 & 0.011 \\
\hline DI & 707.709 & 0.082 & 0.526 & 0.032 & 0.480 & - & 0.472 & - & 0.025 & 0.012 \\
\hline DI & 721.756 & 0.220 & 0.564 & 0.031 & 0.508 & - & 0.513 & - & 0.026 & 0.013 \\
\hline
\end{tabular}

$1 \mathrm{D}$ and $3 \mathrm{D}$ models, the $3 \mathrm{D}$ correction defined by Caffau \& Ludwig (2007) as $A(\mathrm{X})_{3 \mathrm{D}}-A(\mathrm{X})_{1 \mathrm{D}_{\mathrm{LHD}}}$, and the difference between $3 \mathrm{D}$ and $\langle 3 \mathrm{D}\rangle$ models. We report also the error $\left(\sigma_{E W}\right)$ in the Eu abundance due to the uncertainty in the $E W$ measurement, which we estimated by performing $E W$ measurements using different continuum placements and deblending assumptions, for each line: typically this is $\sim 0.02-0.03 \mathrm{dex}$, with only the Eu II line at $412.972 \mathrm{~nm}$ showing an error in the abundance of $\sim 0.05$ dex, probably due to the blending on the blue side of the spectrum.

To place solar 3D correction results in a wider context, we computed 3D corrections of the $664.5 \mathrm{~nm}$ Eu II line for flux spectra in F and G-type atmospheric stellar models. We explored a parameter grid including $T_{\text {eff }}$ between 4980 and $6460 \mathrm{~K}$, $\log g=3.5,4.0,4.5$ and $[\mathrm{M} / \mathrm{H}]=0.0,-1.0$. The Eu abundance is scaled with respect to the metallicity of the model, according to the solar ratio. The reference solar Eu abundance is 0.52 . The results are listed in Table 3. The majority of the 3D corrections (3D-1 $\mathrm{D}_{\text {LHD }}$ ) are negligible, and the largest is just $0.011 \mathrm{dex}$. The $3 \mathrm{D}$ correction related to the average temperature profile $(3 \mathrm{D}-\langle 3 \mathrm{D}\rangle)$ is in the range $0.01-0.02 \mathrm{dex}$ for all models and it is larger than the complete $3 \mathrm{D}$ correction.

As an additional check to test the consistency of our results, we performed a classical 1D analysis on these 5 features. This step is necessary to compare the results obtained by the LHD models and the 1D models, which are usually used in the chemical analysis. To compute the abundance, we used line-profile fitting and employed the line list from the Kurucz database, updated including the atomic parameters for the Eu II lines. This was completed using a code (Caffau et al. 2005) that performs a linear interpolation in a syntheticspectra grid with the Eu abundance as a free-fitting parameter: the final best-fit was derived using numerical $\chi^{2}$ minimisation, using MINUIT (James 1998). Even the line-shift and the continuum-placement can be a free parameter to optimise the fit. Only for two strong features, namely 412.9 and $664.5 \mathrm{~nm}$, we adopted a different version of this code, which include as free-fitting parameters both the Eu abundance and the fraction of the $\mathrm{Eu}$ isotope ${ }^{151} \mathrm{Eu}$ with respect to the total abundance, $\log \left(N\left({ }^{151} \mathrm{Eu}\right) / N\left(\mathrm{Eu}_{\mathrm{tot}}\right)\right)$.
Table 3. The $\mathrm{CO}^{5} \mathrm{BOLD}$ models considered in this work (excluding the solar model) for the Eu II spectral line at $664.519 \mathrm{~nm}$ : the table reports the atmospheric parameters $\left(T_{\text {eff }} / \log g /[\mathrm{M} / \mathrm{H}]\right)$ for each model, the $E W$ measurement, and the corresponding $3 \mathrm{D}-1 \mathrm{D}_{\mathrm{LHD}}$ and $3 \mathrm{D}-\langle 3 \mathrm{D}\rangle$ corrections.

\begin{tabular}{lccc}
\hline \hline $\begin{array}{l}\text { Model parameters } \\
\left(T_{\text {eff }} / \log g /[\mathrm{M} / \mathrm{H}]\right)\end{array}$ & $\begin{array}{c}E W \\
(\mathrm{pm})\end{array}$ & $\begin{array}{c}3 \mathrm{D}-1 \mathrm{D}_{\text {LHD }} \\
(\mathrm{dex})\end{array}$ & $\begin{array}{c}3 \mathrm{D}-\langle 3 \mathrm{D}\rangle \\
(\mathrm{dex})\end{array}$ \\
\hline $5430 / 3.5 / 0.0$ & 1.070 & -0.001 & 0.007 \\
$5480 / 3.5 /-1.0$ & 0.190 & 0.007 & 0.019 \\
$5930 / 4.0 / 0.0$ & 0.670 & 0.004 & 0.008 \\
$5850 / 4.0 /-1.0$ & 0.110 & 0.009 & 0.023 \\
$4980 / 4.5 / 0.0$ & 0.400 & 0.000 & 0.009 \\
$5060 / 4.5 /-1.0$ & 0.073 & -0.003 & 0.009 \\
$5870 / 4.5 / 0.0$ & 0.430 & 0.006 & 0.015 \\
$5929 / 4.5 /-1.0$ & 0.066 & 0.011 & 0.021 \\
$6230 / 4.5 / 0.0$ & 0.400 & 0.009 & 0.017 \\
$6240 / 4.5 /-1.0$ & 0.058 & 0.008 & 0.017 \\
$6460 / 4.5 / 0.0$ & 0.370 & 0.008 & 0.015 \\
$6460 / 4.5 /-1.0$ & 0.051 & 0.001 & 0.013 \\
\hline
\end{tabular}

\section{Results and discussion}

The main results of this analysis are:

1. The 3D analysis, based on different high-resolution high signal-to-noise solar spectra and by using the $\mathrm{CO}^{5} \mathrm{BOLD}$ model, provided a mean $\mathrm{Eu}$ photospheric abundance of $A(\mathrm{Eu})=0.506$ dex with a standard deviation $\sigma=0.008$ for the flux spectra, using the first three spectral features, and $A(\mathrm{Eu})=0.527$ with $\sigma=0.024$ for the intensity spectra at the disk-centre, using all the five lines. As a final value of Eu solar photospheric abundance we recommend $A(\mathrm{Eu})=0.518 \mathrm{dex}(\sigma=0.024)$. This value comes from the average of all measurements, both flux and intensity, since they are very close, and is consistent with the previous $1 \mathrm{D}$ determinations.

2. The difference $3 \mathrm{D}-\langle 3 \mathrm{D}\rangle$ allows us to estimate the $3 \mathrm{D}$ corrections due to the horizontal temperature fluctuations, a component which was not taken into account in the classical 1D models. This correction is negligible for all of the lines considered, with an average value of $-0.009 \operatorname{dex}(\sigma=0.016)$ 
Table 4. Linelist for the five selected Eu II transitions: $\log g f$, excitation potential and corresponding isotope for each hyperfine component are reported.

\begin{tabular}{|c|c|c|c|c|c|c|c|c|c|c|c|}
\hline $\begin{array}{c}\text { Wavelength } \\
\text { (nm) }\end{array}$ & $\overline{\log g f}$ & $\begin{array}{l}E . P . \\
(\mathrm{eV})\end{array}$ & Isotope & $\begin{array}{c}\text { Wavelength } \\
(\mathrm{nm})\end{array}$ & $\overline{\log g f}$ & $\begin{array}{l}E . P . \\
(\mathrm{eV})\end{array}$ & Isotope & $\begin{array}{c}\text { Wavelength } \\
(\mathrm{nm})\end{array}$ & $\overline{\log g f}$ & $\begin{array}{l}E . P . \\
(\mathrm{eV})\end{array}$ & Isotope \\
\hline 412.9627 & -1.34 & 0.000 & 151 & 604.9532 & -2.36 & 1.278 & 151 & 664.5149 & -1.22 & 1.379 & 151 \\
\hline 412.9623 & -1.81 & 0.000 & 151 & 604.9531 & -2.83 & 1.278 & 151 & 664.5142 & -2.13 & 1.379 & 151 \\
\hline 412.9649 & -1.81 & 0.000 & 151 & 604.9529 & -2.83 & 1.278 & 151 & 664.5133 & -3.55 & 1.379 & 151 \\
\hline 412.9645 & -1.28 & 0.000 & 151 & 604.9528 & -2.30 & 1.278 & 151 & 664.5136 & -1.14 & 1.379 & 151 \\
\hline 412.9640 & -1.62 & 0.000 & 151 & 604.9526 & -2.64 & 1.278 & 151 & 664.5127 & -1.94 & 1.379 & 151 \\
\hline 412.9676 & -1.62 & 0.000 & 151 & 604.9524 & -2.64 & 1.278 & 151 & 664.5116 & -3.38 & 1.379 & 151 \\
\hline 412.9671 & -1.15 & 0.000 & 151 & 604.9522 & -2.17 & 1.278 & 151 & 664.5120 & -1.06 & 1.379 & 151 \\
\hline 412.9665 & -1.56 & 0.000 & 151 & 604.9519 & -2.58 & 1.278 & 151 & 664.5108 & -1.88 & 1.379 & 151 \\
\hline 412.9710 & -1.56 & 0.000 & 151 & 604.9516 & -2.58 & 1.278 & 151 & 664.5094 & -3.45 & 1.379 & 151 \\
\hline 412.9704 & -1.00 & 0.000 & 151 & 604.9514 & -2.02 & 1.278 & 151 & 664.5101 & -0.97 & 1.379 & 151 \\
\hline 412.9698 & -1.59 & 0.000 & 151 & 604.9510 & -2.62 & 1.278 & 151 & 664.5087 & -1.93 & 1.379 & 151 \\
\hline 412.9753 & -1.59 & 0.000 & 151 & 604.9506 & -2.62 & 1.278 & 151 & 664.5070 & -3.77 & 1.379 & 151 \\
\hline 412.9746 & -0.85 & 0.000 & 151 & 604.9502 & -1.87 & 1.278 & 151 & 664.5079 & -0.89 & 1.379 & 151 \\
\hline 412.9739 & -1.78 & 0.000 & 151 & 604.9496 & -2.80 & 1.278 & 151 & 664.5062 & -2.12 & 1.379 & 151 \\
\hline 412.9803 & -1.78 & 0.000 & 151 & 604.9492 & -2.80 & 1.278 & 151 & 664.5056 & -0.82 & 1.379 & 151 \\
\hline 412.9796 & -0.70 & 0.000 & 151 & 604.9486 & -1.72 & 1.278 & 151 & 664.5123 & -1.22 & 1.379 & 153 \\
\hline 412.9681 & -1.34 & 0.000 & 153 & 604.9514 & -2.36 & 1.278 & 153 & 664.5121 & -2.13 & 1.379 & 153 \\
\hline 412.9678 & -1.81 & 0.000 & 153 & 604.9515 & -2.83 & 1.278 & 153 & 664.5118 & -3.55 & 1.379 & 153 \\
\hline 412.9690 & -1.81 & 0.000 & 153 & 604.9514 & -2.83 & 1.278 & 153 & 664.5115 & -1.14 & 1.379 & 153 \\
\hline 412.9688 & -1.28 & 0.000 & 153 & 604.9515 & -2.30 & 1.278 & 153 & 664.5112 & -1.94 & 1.379 & 153 \\
\hline 412.9684 & -1.62 & 0.000 & 153 & 604.9515 & -2.64 & 1.278 & 153 & 664.5107 & -3.38 & 1.379 & 153 \\
\hline 412.9701 & -1.62 & 0.000 & 153 & 604.9515 & -2.64 & 1.278 & 153 & 664.5106 & -1.06 & 1.379 & 153 \\
\hline 412.9698 & -1.15 & 0.000 & 153 & 604.9515 & -2.17 & 1.278 & 153 & 664.5101 & -1.88 & 1.379 & 153 \\
\hline 412.9694 & -1.56 & 0.000 & 153 & 604.9515 & -2.58 & 1.278 & 153 & 664.5095 & -3.45 & 1.379 & 153 \\
\hline 412.9715 & -1.56 & 0.000 & 153 & 604.9514 & -2.58 & 1.278 & 153 & 664.5097 & -0.97 & 1.379 & 153 \\
\hline 412.9712 & -1.00 & 0.000 & 153 & 604.9513 & -2.02 & 1.278 & 153 & 664.5090 & -1.93 & 1.379 & 153 \\
\hline 412.9709 & -1.59 & 0.000 & 153 & 604.9511 & -2.62 & 1.278 & 153 & 664.5081 & -3.77 & 1.379 & 153 \\
\hline 412.9733 & -1.59 & 0.000 & 153 & 604.9510 & -2.62 & 1.278 & 153 & 664.5088 & -0.89 & 1.379 & 153 \\
\hline 412.9731 & -0.85 & 0.000 & 153 & 604.9508 & -1.87 & 1.278 & 153 & 664.5079 & -2.12 & 1.379 & 153 \\
\hline 412.9730 & -1.78 & 0.000 & 153 & 604.9503 & -2.80 & 1.278 & 153 & 664.5081 & -0.82 & 1.379 & 153 \\
\hline 412.9756 & -1.78 & 0.000 & 153 & 604.9500 & -2.80 & 1.278 & 153 & & & & \\
\hline 412.9755 & -0.70 & 0.000 & 153 & 604.9496 & -1.72 & 1.278 & 153 & & & & \\
\hline $\begin{array}{c}\text { Wavelength } \\
\text { (nm) }\end{array}$ & $\overline{\log g f}$ & $\begin{array}{l}E . P . \\
(\mathrm{eV})\end{array}$ & Isotope & $\begin{array}{c}\text { Wavelength } \\
(\mathrm{nm})\end{array}$ & $\overline{\log g f}$ & $\begin{array}{l}E . P . \\
(\mathrm{eV})\end{array}$ & Isotope & $\begin{array}{c}\text { Wavelength } \\
(\mathrm{nm})\end{array}$ & $\overline{\log g f}$ & $\begin{array}{l}E . P . \\
(\mathrm{eV})\end{array}$ & Isotope \\
\hline 707.7169 & -2.34 & 1.249 & 151 & 721.7564 & -2.08 & 1.229 & 151 & & & & \\
\hline 707.7164 & -2.64 & 1.249 & 151 & 721.7573 & -2.18 & 1.229 & 151 & & & & \\
\hline 707.7154 & -2.17 & 1.249 & 151 & 721.7549 & -2.63 & 1.229 & 151 & & & & \\
\hline 707.7156 & -3.60 & 1.249 & 151 & 721.7559 & -1.92 & 1.229 & 151 & & & & \\
\hline 707.7146 & -2.46 & 1.249 & 151 & 721.7574 & -1.81 & 1.229 & 151 & & & & \\
\hline 707.7132 & -2.00 & 1.249 & 151 & 721.7534 & -2.59 & 1.229 & 151 & & & & \\
\hline 707.7134 & -3.49 & 1.249 & 151 & 721.7550 & -1.83 & 1.229 & 151 & & & & \\
\hline 707.7120 & -2.40 & 1.249 & 151 & 721.7570 & -1.56 & 1.229 & 151 & & & & \\
\hline 707.7104 & -1.86 & 1.249 & 151 & 721.7517 & -2.74 & 1.229 & 151 & & & & \\
\hline 707.7104 & -3.60 & 1.249 & 151 & 721.7537 & -1.84 & 1.229 & 151 & & & & \\
\hline 707.7086 & -2.44 & 1.249 & 151 & 721.7564 & -1.36 & 1.229 & 151 & & & & \\
\hline 707.7066 & -1.73 & 1.249 & 151 & 721.7496 & -3.10 & 1.229 & 151 & & & & \\
\hline 707.7063 & -3.94 & 1.249 & 151 & 721.7523 & -2.01 & 1.229 & 151 & & & & \\
\hline 707.7045 & -2.62 & 1.249 & 151 & 721.7554 & -1.20 & 1.229 & 151 & & & & \\
\hline 707.7024 & -1.61 & 1.249 & 151 & 721.7605 & -2.08 & 1.229 & 153 & & & & \\
\hline 707.7125 & -2.34 & 1.249 & 153 & 721.7602 & -2.18 & 1.229 & 153 & & & & \\
\hline 707.7125 & -2.64 & 1.249 & 153 & 721.7602 & -2.63 & 1.229 & 153 & & & & \\
\hline 707.7116 & -2.17 & 1.249 & 153 & 721.7598 & -1.92 & 1.229 & 153 & & & & \\
\hline 707.7125 & -3.60 & 1.249 & 153 & 721.7594 & -1.81 & 1.229 & 153 & & & & \\
\hline 707.7115 & -2.46 & 1.249 & 153 & 721.7590 & -2.59 & 1.229 & 153 & & & & \\
\hline 707.7106 & -2.00 & 1.249 & 153 & 721.7586 & -1.83 & 1.229 & 153 & & & & \\
\hline 707.7112 & -3.49 & 1.249 & 153 & 721.7578 & -1.56 & 1.229 & 153 & & & & \\
\hline 707.7104 & -2.40 & 1.249 & 153 & 721.7572 & -2.74 & 1.229 & 153 & & & & \\
\hline 707.7093 & -1.86 & 1.249 & 153 & 721.7564 & -1.84 & 1.229 & 153 & & & & \\
\hline 707.7095 & -3.60 & 1.249 & 153 & 721.7553 & -1.36 & 1.229 & 153 & & & & \\
\hline 707.7086 & -2.44 & 1.249 & 153 & 721.7543 & -3.10 & 1.229 & 153 & & & & \\
\hline 707.7078 & -1.73 & 1.249 & 153 & 721.7531 & -2.01 & 1.229 & 153 & & & & \\
\hline 707.7071 & -3.94 & 1.249 & 153 & 721.7514 & -1.20 & 1.229 & 153 & & & & \\
\hline 707.7063 & -2.62 & 1.249 & 153 & & & & & & & & \\
\hline 707.7060 & -1.61 & 1.249 & 153 & & & & & & & & \\
\hline
\end{tabular}


and $0.010 \mathrm{dex}(\sigma=0.003)$ for flux and intensity, respectively. This difference between the two solar data-sets was already observed in a previous 3D analysis for sulphur (Caffau et al. 2005) and phosphorus (Caffau \& Ludwig 2007), and can be attributed to the different atmospheric layers where intensity and flux spectra originate: the centre disc intensity spectra arise from deeper layers, where the temperature fluctuations are more pronounced.

3. The difference $3 \mathrm{D}-1 \mathrm{D}_{\mathrm{LHD}}$ allows us to compare $3 \mathrm{D}$ and 1D models that use the same physical assumptions, such as equation of state and opacities, and provides a $3 D$ correction. These values are close to zero both in flux and intensity, with an average difference of $0.004 \operatorname{dex}(\sigma=0.013)$ and 0.021 dex $(\sigma=0.004)$ respectively. The $3 \mathrm{D}-1 \mathrm{D}_{\mathrm{LHD}}$ corrections appear to be systematically higher than the $3 \mathrm{D}-\langle 3 \mathrm{D}\rangle$ corrections with a difference of about 0.010 dex.

4. Finally, as consistency check, we performed a classical 1D analysis, using the Holweger \& Müller (1974) and ATLAS models. We derived a mean photospheric abundance for europium of $A(\mathrm{Eu})=0.515 \mathrm{dex}(\sigma=0.022)$ and $0.523 \mathrm{dex}(\sigma=0.014)$ for disc-centre intensity and flux, respectively, in good agreement with the previous values of Anders \& Grevesse (1989) of 0.51 dex and Lawler et al. (2001) of 0.52 dex. The isotopic ratio $\left(N\left({ }^{151} \mathrm{Eu}\right) / N\left(\mathrm{Eu}_{\mathrm{tot}}\right)\right)$ computed using the two strongest Eu lines agrees well with the meteoritic ratio: $0.49(\sigma=0.023)$ and $0.50(\sigma=0.023)$ for intensity and flux, respectively.

There is no way to know a priori if 3D effects are important for any given line. A detailed calculation has to be done in each case. Solar abundances are widely used as a reference and their implication goes beyond pure chemical composition, but touches fields such as helioseismology and solar-neutrino production. The low solar abundances measured by Asplund et al. (2005) have put some strain on our understanding of both. As suggested by Bahcall et al. (2005), different measurements of solar abundances, obtained using different observed spectra and different solar models, allow a better estimation of the systematic uncertainties. In the case of $\mathrm{Eu}$, we conclude that the $3 \mathrm{D}$ effects are negligible in the Sun and solar-like stars. The scenario is very coherent, when we consider 3D of the non-solar models. 3D corrections are negligible for both solar and slightly metal-poor models. This is in line with the findings discussed by Steffen \& Holweger (2002), which investigated granulation corrections in the Sun for several elements. Although Eu is not included in this study, we can compare our results for Eu with their findings for Sr. These two elements show very similar line-formation properties for spectral lines with similar excitation potential and oscillator strength. Also for $\mathrm{Sr}$, the corrections are negligible, typically between -0.02 and +0.02 . Therefore, for $\mathrm{Eu}$, as for $\mathrm{S}$ (Caffau et al. 2007a) and P (Caffau et al. 2007b) we conclude that the use of 3D models does not imply a substantial downward revision of the solar abundances with respect to that deduced using 1D models.
Acknowledgements. We warmly thank the referee, A. J. Sauval, for his useful suggestions. The authors E.C., H.-G.L., P.B. acknowledge financial support from EU contract MEXT-CT-2004-014265 (CIFIST). We acknowledge use of the supercomputing centre CINECA, which has granted us time to compute part of the hydrodynamical models used in this investigation, through the INAF-CINECA agreement 2006, 2007.

\section{References}

Anders, E., \& Grevesse, N. 1989, Geochim. Cosmochim. Acta, 53, 197 Arlandini, C., Kappeler, F., Wisshak, K., et al. 1999, ApJ, 525, 886

Asplund, M., Grevesse, N., \& Sauval, A. J. 2005, Cosmic Abundances as Records of Stellar Evolution and Nucleosynthesis, ASP Conf. Ser., 336, 25 Bahcall, J. N., Basu, S., \& Serenelli, A. M. 2005, ApJ, 631, 1281

Biemont, E., Karner, C., Meyer, G., Traeger, F., \& zu Putlitz, G. 1982, A\&A, 107,166

Bonifacio, P. 2005, Mem. Soc. Astron. It. Suppl., 8, 114

Bonifacio, P., Hill, V., Molaro, P., et al. 2000, A\&A, 359, 663

Burbidge, E. M., Burbidge, R. R., Fowler, W. A., \& Hoyle, F. 1957, Rev. Mod. Phys., 29, 547

Burris, D. L., Pilachowski, C. A., Armandroff, T. E., et al. 2000, ApJ, 544, 302

Caffau, E., \& Ludwig, H.-G. 2007, A\&A, 467, L11

Caffau, E., Bonifacio, P., Faraggiana, R., et al. 2005, A\&A, 441, 533

Caffau, E., Faraggiana, R., Bonifacio, P., Ludwig, H.-G., \& Steffen, M. 2007a, A\&A, 470, 699

Caffau, E., Steffen, M., Sbordone, L., Ludwig, H.-G., \& Bonifacio, P. 2007b, A\&A, 473, L9

Cayrel, R., Spite, M., Spite, F., et al. 1999, A\&A, 343, 923

Cowan, J. J., \& Sneden, C. 2004, Carnegie Observatories Astrophysics Series, 4, ed. A. McWilliam, \& M. Rauch (Cambridge Univ. Press), 27

Delbouille, L., Roland, G., \& Neven, L. 1973, Photometric Atlas of the Solar Spectrum from $\lambda \lambda 3000$ to $\lambda \lambda 10000$ (Liège: Univ. Liège, Institut d'Astrophysique)

Freytag, B., Steffen, M., \& Dorch, B. 2002, Astron. Nachr., 323, 213

Grevesse, N., \& Sauval, A. J. 1998, SSRv, 85, 161

Hill, V. 1997, A\&A, 324, 435

Hill, V., Andrievsky, S., \& Spite, M. 1995, A\&A, 293, 347

Holweger, H., \& Müller, E. A. 1974, Sol. Phys., 39, 19

James, F. 1998, MINUIT, Reference Manual, Version 94.1, CERN, Geneva, Switzerland

Lawler, J. E., Wickliffe, M. E., Den Hartog, E. A., \& Sneden, C. 2001, ApJ, 563, 1075

Kurucz, R. 1993, SYNTHE Spectrum Synthesis Programs and Line Data. Kurucz CD-ROM No. 18 (Cambridge, Mass.: Smithsonian Astrophysical Observatory), 18

Kurucz, R. L. 2005, Mem. Soc. Astron. It. Suppl., 8, 14

Mashonkina, L. I. 2000, Astron. Rep., 44, 558

Mashonkina, L., \& Gehren, T. 2000, A\&A, 364, 249

Mihalas, D. 1978, Stellar atmospheres, 2nd edn. (San Francesico: W. H. Freeman and Co.)

Neckel, H., \& Labs, D. 1984, Sol. Phys., 90, 205

Sbordone, L. 2005, Mem. Soc. Astron. It. Suppl., 8, 61

Sbordone, L., Bonifacio, P., Castelli, F., \& Kurucz, R. L. 2004, Mem. Soc. Astron. It. Suppl., 5, 93

Steffen, M., \& Holweger, H. 2002, A\&A, 387, 258

Wahlgren, G. M. 2005, Mem. Soc. Astron. It. Suppl., 8, 108

Wahlgren, G. M., Leckrone, D. S., Johansson, S. G., Rosberg, M., \& Brage, T. 1995, ApJ, 444, 438

Wedemeyer, S., Freytag, B., Steffen, M., Ludwig, H.-G., \& Holweger, H. 2004, A\&A, 414, 1121 